\title{
Theoretical Investigation into Spectral Coexistence of CDMA and TDMA Systems
}

\author{
A.M. Abbosh ${ }^{* 1}$ and M. A. Shehathah² \\ ${ }^{1}$ School of ITEE, The University of Queensland, Brisbane Q4072, Australia \\ ${ }^{2}$ Department of Electronics Engineering, University of Mosul, Iraq \\ Received 22 November 2004; accepted 11 October 2006
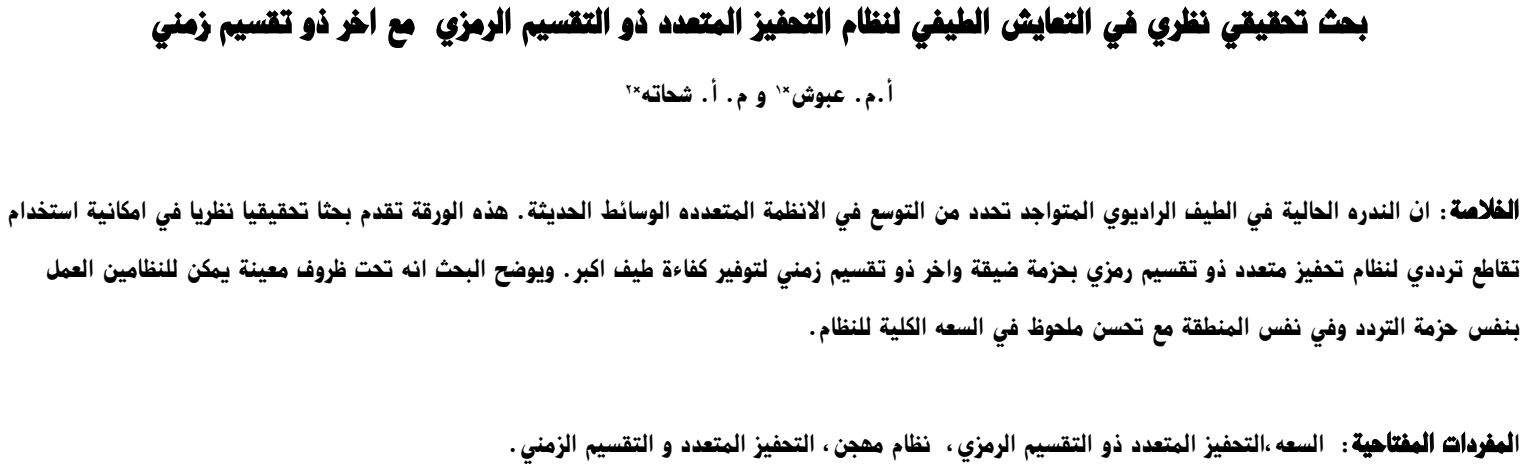

\begin{abstract}
The scarcity of available radio spectrum presently limits the extension of modern multimedia systems. This paper presents a theoretical investigation into the possibility of using a frequency overlay of a narrowband Code Division Multiple Access (CDMA) System and a Time Division Multiple Access (TDMA) System to provide a greater spectral efficiency. This paper shows that under certain conditions the two systems can operate in the same frequency band and in the same area with a considerable improvement in the overall capacity of the whole system.
\end{abstract}

Keywords: Capacity, CDMA, Hybrid system, TDMA

\section{Introduction}

Given the constraints of the high cost and limited availability of radio spectrum, efficient spectrum usage is a cornerstone in the economic success of third generation cellular systems. One novel approach to using available resources more efficiently is the combination of existing radio systems into coordinated, hybrid systems, in order to combine the strength and capabilities of the individual systems. The combination of systems adds a degree of freedom in the sense that at any time the most spectrally efficient transport system can be chosen depending for example on quality-of-service requirements and traffic characteristics.

Most of the third generation systems use CDMA as the multiple access method. Furthermore some systems use TDMA or FDMA or both as the multiple access method. CDMA systems use spreading methods to spread a narrow band signal into a wideband signal. Since the spreading process does not change the power of the signal, the power spectral density of the spread signal is lower than the power density of the narrowband signal. Figure 1 shows the principle of the spreading and de-spreading process. The narrow band useful signal is spread and the resulting signal is transmitted over a wideband channel

*Corresponding author’s e-mail: abbosh@itte.uq.edu.au and de-spread in the receiver. As can be seen in Fig. 1, the de-spreading process in the CDMA receiver operates as a spreading process for a narrowband interfering signals. In terms of coexistence of TDMA/FDMA and CDMA systems, the narrow band TDMA (or FDMA) channels act as narrowband interferer for the CDMA system if they exist in the same frequency band as the CDMA carrier. From the TDMA (or FDMA) systems point of view, the CDMA system acts like a noise-like interferer with a low power spectral density. The receiver filter in the TDMA (or FDMA) receiver will make sure that the noise-like interfering signal will only be received in the narrow bandwidth of the TDMA system. Thus, only a small part of the interference power originating in the CDMA system will be received, see Fig. 2.

The need for future multiple access schemes employing an overlay is stressed in many papers (Schilling, Lomp and Garodnick, 1993; Grieco and Schilling, 1994; Himimy and Gupta, 1996; Widdowson, 1997; Koorevaar and Ruprecht, 1999). In all these papers simulation algorithms were used to study the effects of overlaying different schemes on the performance of the hybrid systems. In addition to simulation, some authors tried to study the overlay process from a theoretical point of view (Singh, 1997; Latchman and Chitamu, 2003). The inaccuracy of the model used due to oversimplification of the assumptions leads to incorrect expectations. 


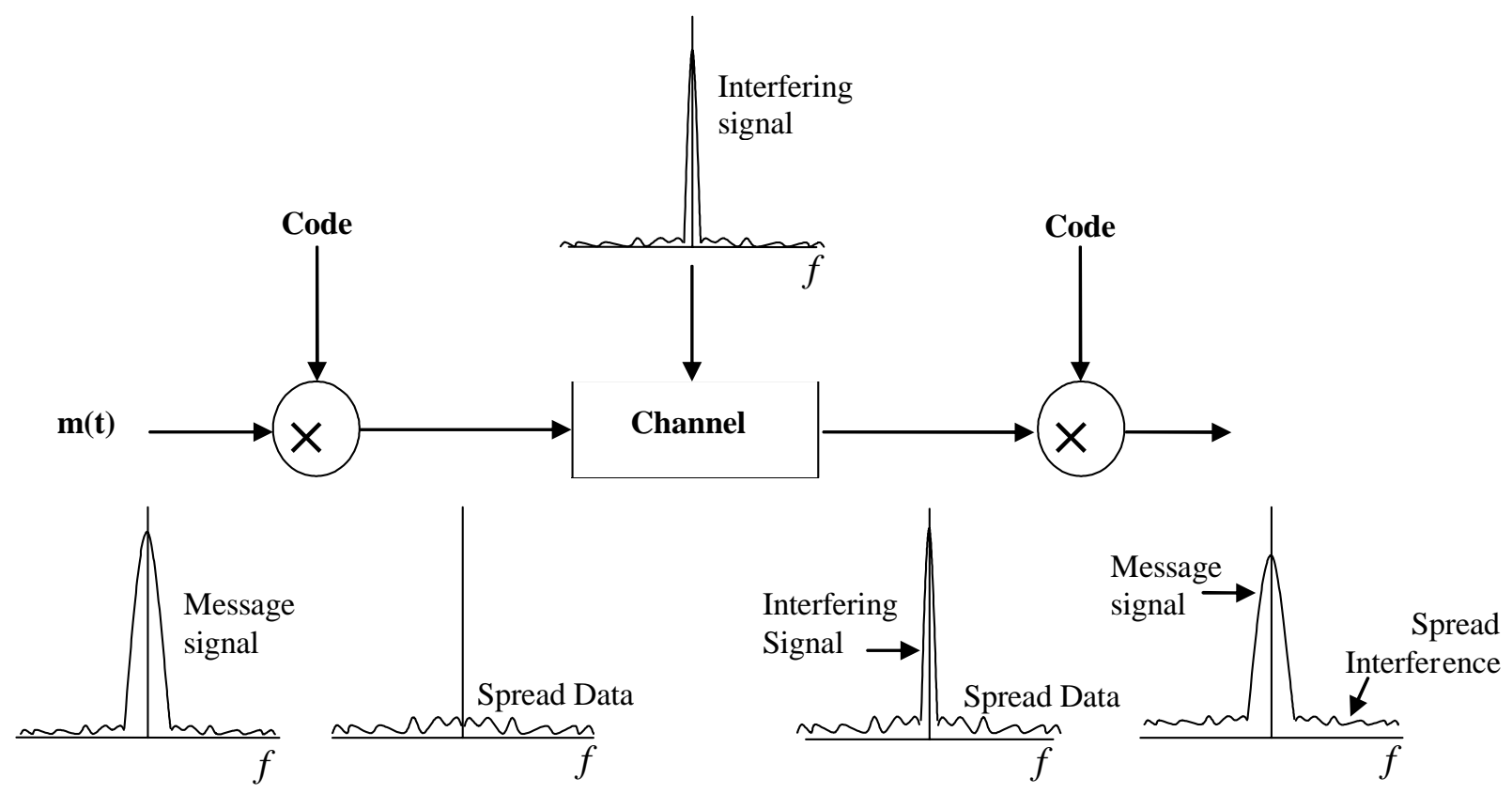

Figure 1. Effect of the TDMA signal on the CMDA receiver

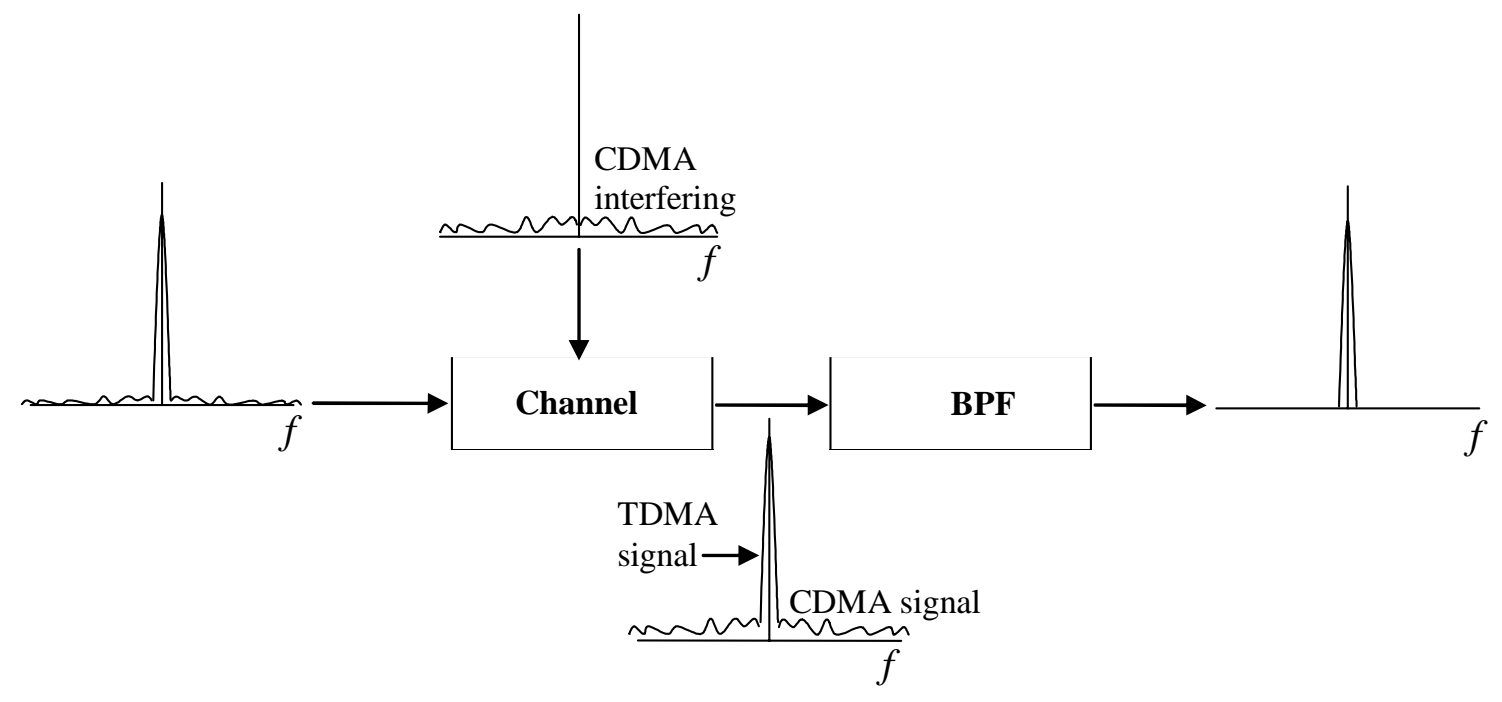

Figure 2. Effect of the CMDA signal on the TDMA receiver

In this paper, a Fourier transform is used to investigate the benefits of spectral coexistence of CDMA and TDMA systems. Also the model used in this paper considers the case of complete overlapping between the two systems to explore the worst case condition. Effects of the voice activity ratio, the TDMA duty cycle and the code used in CDMA system besides the lack of synchronization between CDMA and TDMA are included in the analysis.

\section{Analysis}

The analysis considers the mutual effect of CDMA and TDMA systems operating in the same area and using the same frequency band. The overall capacity of the hybrid system is investigated to see whether the perform- ance is degraded or enhanced. In the following analysis the capacity of the system is assumed to be the total number of users working at the same time with a signal to noise ratio above the threshold level.

\subsection{Effect of the CDMA Signal on the TDMA Receiver}

Assume a CDMA signal of the form:

$$
r(t)=\sqrt{2 P_{C}} c(t) m(t) \cos \left(w_{C} t\right)
$$

where $P_{c}$ is the CDMA received power at the base station, $m(t)$ is the message signal, $c(t)$ is the chip sequence, $w_{c}$ is the radian carrier frequency. 
Let us assume that: $T_{b}$ is the bit duration, $T_{c}$ is the chip duration, $k_{1}=T_{b} / T_{c}$ is the number of chips per bit, $C(f)$ is the Fourier transform (F.T.) of $c(t), M(f)$ is F.T. of $m(t)$ and $R(f)$ is F.T. of $r(t)$. As $k_{1}>>1$ according to definition of CDMA then $M(f)$ appears as a Dirac delta function to the relatively wideband $C(f)$ spectrum. For a single bit of duration $T_{b}$ then $R(f)$ is:

$$
R(f)=\sqrt{\frac{P_{C}}{2}}\left[C_{T b}\left(f+f_{c}\right)+C_{T b}\left(f-f_{c}\right)\right]
$$

where $C_{T b}(f)$ is the F.T of the k-chips in a single bit duration $T_{b}$ which is given by:

$$
C_{T b}(f)=T_{c} \operatorname{sinc}\left(f T_{c}\right) \sum_{i=1}^{k_{1}} u_{i} e^{-j \pi f(2 i-1) T c}
$$

where $u_{i}= \pm 1$ and $\operatorname{sinc}(\mathrm{x})=\sin (\mu \mathrm{x}) / \mu \mathrm{x}$.

The amount of the CDMA interference energy experienced in a bandwidth equal to $B$ by a TDMA signal working at carrier frequency $f_{t}$, is given as:

$$
E_{i}(f)=\int_{f_{t}-B / 2}^{f}|R(f)|^{2} d f
$$

Substituting from (2) into (4);

$$
E_{i}(f)=2 \frac{P_{C}}{2} \int_{f_{t}-\frac{B}{2}}^{f}\left|T_{C} \operatorname{sinc}\left(\frac{f-f_{C}}{f_{C}}\right) \sum_{i=1}^{k_{1}} u_{i} e^{-j \pi\left(f-f_{C}\right)(2 i-1) T_{C}}\right|^{2} d f
$$

Since $f$ varies from $f_{t}-B / 2$ to $f_{t}+B / 2$ (as maximum), and assuming $f_{t}=f_{c}$ (full overlaying of CDMA \& TDMA); hence: $f-f_{t}=\left(f_{c} \pm B / 2\right)-f_{t}= \pm B / 2$. As $\pm B / 2$ is very small relative to $f_{c}$, we can assume that $\sin c\left( \pm B / 2 f_{c}=\right.$ 1. Hence (5) can be written as:

$$
E_{i}(f)=\frac{P_{c}}{f_{c}^{2}} \int_{f_{t}-\frac{B}{2}}^{f}\left|\sum_{i=1}^{k_{1}} u_{i} e^{-j \pi\left(f-f_{c}\right)(2 i-1) T_{C}}\right|^{2} d f
$$

Therefore, the interference power from the CDMA source in that small bandwidth $(B)$ can be expressed as:

$$
P_{c i}=\int_{f_{t}-\frac{B}{2}}^{f_{t}+\frac{B}{2}} E_{i}(f) d f
$$

Substituting (6) into (7);

$P_{c i}=\frac{P_{c}}{f_{c}^{2}} \int_{f_{t}-\frac{B}{2}}^{f_{t}+\frac{B}{2}} \quad \int_{f_{t}-\frac{B}{2}}^{f}\left|\sum_{i=1}^{k_{1}} u_{i} e^{-j \pi\left(f-f_{c}\right)(2 i-1) T_{c}}\right|^{2} d f d f(8)$ is:

Assuming that the result of the double integral of (8)

$$
P_{c i}=g_{1} P_{c}
$$

where $g_{1}$ is a constant that depends on the bandwidth (B) and code type and length. The values of $g_{1}$ has been calculated numerically using MATLAB program and Walsh code. Depending on the parameter values shown in Table 1 , the value of $g_{1}$ is shown to be equal to 0.0021 .

The signal to noise ratio at the TDMA receiver $\left(S_{t}\right)$ can be expressed as:

$$
S_{t}=\frac{P_{t}}{N_{1} v g_{1} P_{c}+N_{o}}
$$

Where $P_{t}$ is TDMA received power, $N_{1}=$ number of CDMA users, $v=$ voice activity ratio $(\leq 0.5), N_{O}=$ Thermal noise $=K T B$; where $K=$ Boltzmann's constant $=1.38 * 10^{-23}$ Joules/Kelvin, $T$ is the temperature in Kelvin.

\subsection{Effect of the TDMA Signal on the CDMA Receiver}

Let the TDMA signal be represented by:

$$
g(t)=\sqrt{2 P_{t}} b(t) \cos \left(w_{t} t\right)
$$

where $b(t)$ is the information signal (message). At the CDMA receiver, the TDMA signal appears as:

$$
(x)=g(t) c(t)
$$

Substituting (11) into (12);

$$
x(t)=\sqrt{2 P_{t}} b(t) c(t) \cos \left(w_{t} t\right)
$$

Let $T_{d}$ be the bit duration for the TDMA signal, $k_{d}=$ integer $\left(T_{d} / T_{c}\right)=$ number of chips per bit.

For a single bit of duration, $T_{d}$, F.T of $x(t)$ is:

$$
X(f)=\sqrt{\frac{P_{t}}{2}}\left[C_{T d}\left(f+f_{t}\right)+C_{T d}\left(f-f_{t}\right)\right]
$$

$C_{T d}(f)$ is F.T of $k_{2}$ chips in a single bit duration which is given by:

$$
C_{T d}(f)=T_{c} \operatorname{sinc}\left(f T_{c}\right) \sum_{i=1}^{k_{2}} u_{i} e^{-j \pi f(2 i-1) T c}
$$

The amount of the TDMA energy $\left(E_{t}\right)$ actually lying within the passband of the CDMA receiver working at carrier frequency $f_{c}$ can be written as in (6):

$$
E_{i}(f)=\frac{P_{t}}{f_{c}^{2}} \int_{f_{C}-\frac{B}{2}}^{f}\left|\sum_{i=1}^{k_{2}} u_{i} e^{-j \pi\left(f-f_{t}\right)(2 i-1) T_{C}}\right|^{2} d f
$$

The value of the TDMA power interfered with the CDMA signal is: 
Table 1. Values of different parameters used in the paper

$\begin{array}{cccc}\text { Parameter } & \text { Value } & \text { Parameter } & \text { Value } \\ B=1 / T_{b} & 19200 \mathrm{~Hz} & T_{c} & 1 / 1.2288 * 10^{6} \mathrm{~s} \\ S_{t} & 8 & S_{c} & 0.04 \\ P_{t i}=\int_{C}+\frac{B}{2} \\ f_{C}-\frac{B}{2}\end{array}$

As before, the integral above can be assumed to be equal to:

$$
P_{t i}=g_{2} P_{t}
$$

The value of $g_{2}$ can be calculated for all the possible Walsh codes. Figure 3 shows this result. It is to be concluded here that due to lack of synchronization CDMA and TDMA systems, as they work separately, then all possible values of $g_{2}$ are shown in Fig. 3 can be expected. To analyze the worst case condition we shall take $g_{2}$ $=0.00386$, see Fig. 3 .

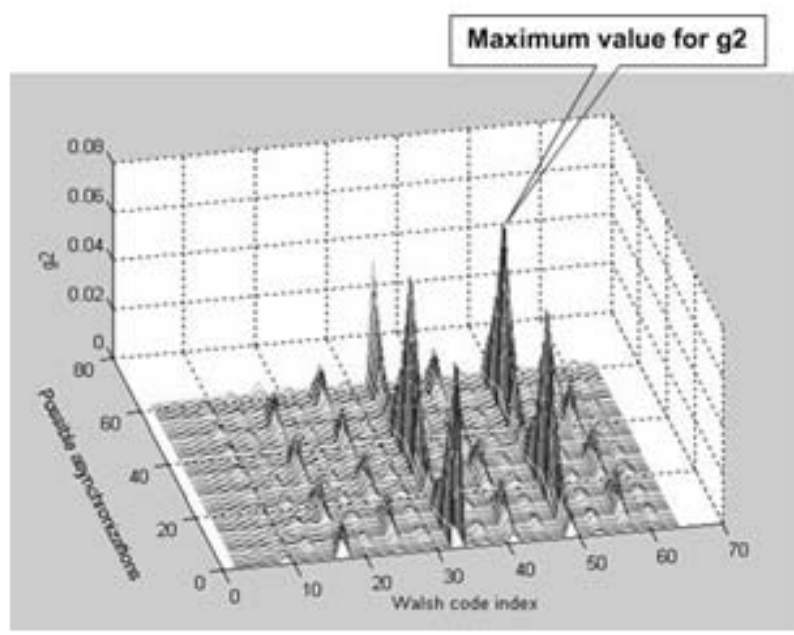

Figure 3. Variation of $g_{2}$ with Walsh code index and possible synchronizations. Maximum value for $g_{2}$ (the worst case scenario) is shown

The signal to noise ratio at the CDMA receiver $\left(S_{c}\right)$ after using (18) can be expressed as:

$$
S_{c}=\frac{P_{c}}{\left(N_{1}-1\right) v P_{C}+N_{2} \theta v g_{2} P_{t}+N_{o}}
$$

where $N_{2}=$ number of TDMA users, $v=$ voice activity ratio $(\leq 0.5), \quad \theta=$ TDMA duty cycle $(\leq 0.125)$.

\section{Capacity of the Hybrid System}

In order to see if the spectral coexistence of CDMA and TDMA systems is useful and possible we have to investigate the variation of the system capacity (CDMA+TDMA) as a function of number of CDMA users and TDMA users for different CDMA received power (or different TDMA received power), this study is developed for constant values of $S_{c}$ and $S_{t}$.

Solving (10) for $P_{t}$ :

$P_{t}=S_{t} N_{1} v g_{1} P_{C}+S_{t} N_{o}$

Substituting in (19) we get:

$S_{C}=\frac{P_{C}}{\left(N_{1}-1\right) v P_{C}+N_{2} \theta v g_{2}\left(S_{t} N_{1} P_{C} v g_{1}+S_{t} N_{o}\right)+N_{o}}$

Solving for $N_{2}$, we get:

$N_{2}=\frac{P_{c}-\left(S_{c}\left(N_{1}-1\right) v P_{c}+S_{c} N_{o}\right)}{S_{C} \theta v g_{2}\left(S_{t} N_{1} P_{c} v g_{1}+S_{t} N_{o}\right)}$

Adding $N_{1}$ to both sides of the above equation we get the overall capacity $N_{T}$ :

$$
N_{T}=\frac{P_{C}-\left(S_{c}\left(N_{1}-1\right) v P_{C}+S_{C} N_{o}\right)}{S_{c} \theta v g_{2}\left(S_{t} N_{1} P_{C} v g_{1}+S_{t} N_{o}\right)}+N_{1}
$$

Solving (21) for $N_{1}$ :

$N_{1}=\frac{P_{C}-\left[S_{C} N_{2} \theta v g_{2} S_{t} N_{o t}+S_{C} N_{o c}-S_{C} v P_{C}\right]}{\left.\left[S_{C} v P_{C}+S_{C} N_{2} \theta v g_{2} S_{t} P_{C} v g_{1}\right)\right]}$

By adding $N_{2}$ to both sides of (24):

$$
N_{T}=\frac{P_{C}-\left[S_{C} N_{2} \theta v g_{2} S_{t} N_{o}+S_{C} N_{o}-S_{c} v P_{C}\right]}{\left.\left[S_{c} v P_{C}+S_{C} N_{2} \theta v g_{2} S_{t} P_{C} v g_{1}\right)\right]}+N_{2}
$$

\section{Results of Analysis}

Depending on the results of the theoretical analysis discussed in the last section, variations of the overall capacity in the hybrid system with different parameters is investigated for the worst case (that is, maximum overlapping; $\left.f_{c}=f_{t}\right)$. Values of the needed parameters are selected as shown in Table (1) to comply with practical systems. Figure 4 shows variations of the overall capacity with number of CDMA users $\left(N_{1}\right)$ for different values of the received CDMA power $\left(P_{c}\right)$. It is shown that for a constant value of $P_{c}$, the overall capacity increases with increasing $N_{1}$ till a certain limit after which any increase in $\quad N_{1}$ causes a sudden drop in the overall capacity. This is because increasing the number of users causes degra- 


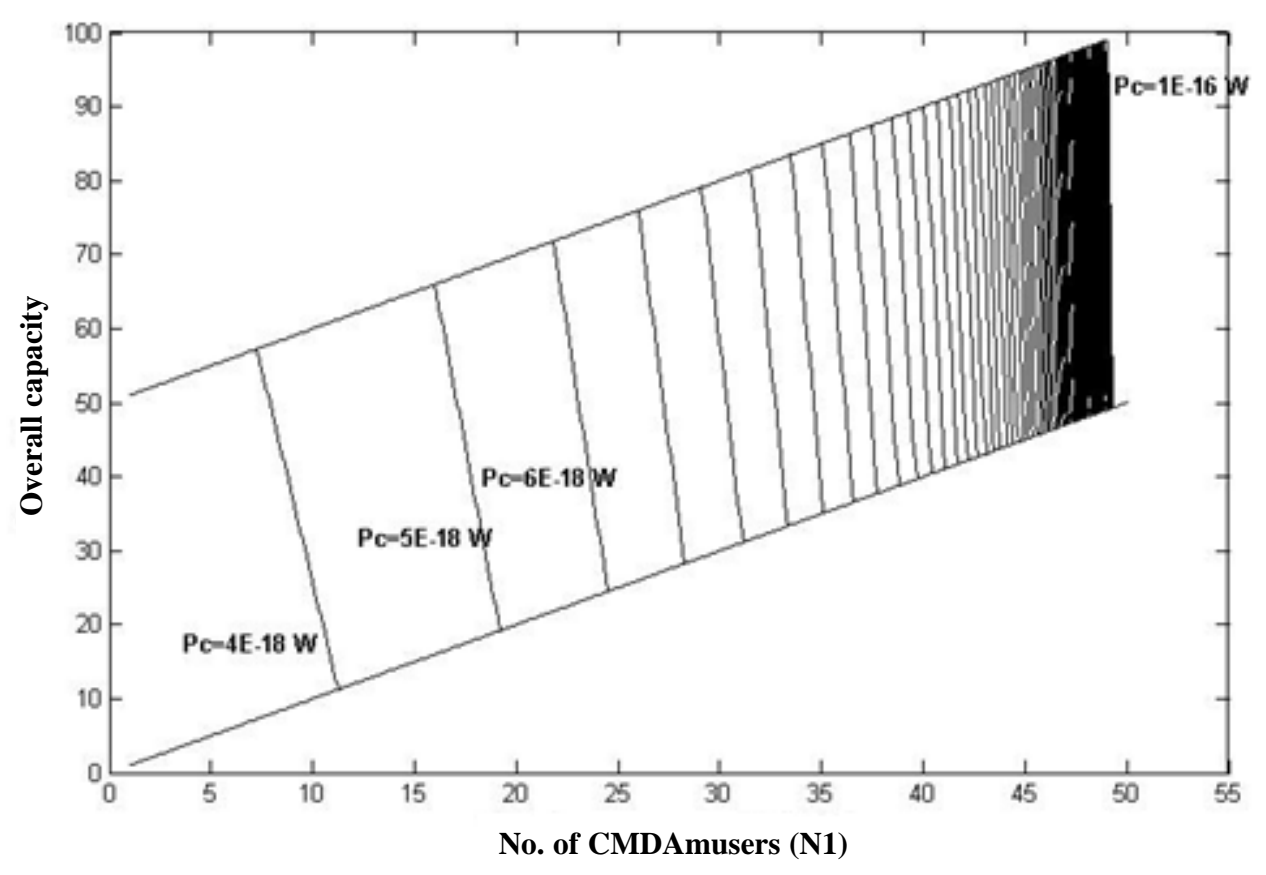

Figure 4. Variation of overall capacity with $N_{1}$ for the different values of $P_{c}$

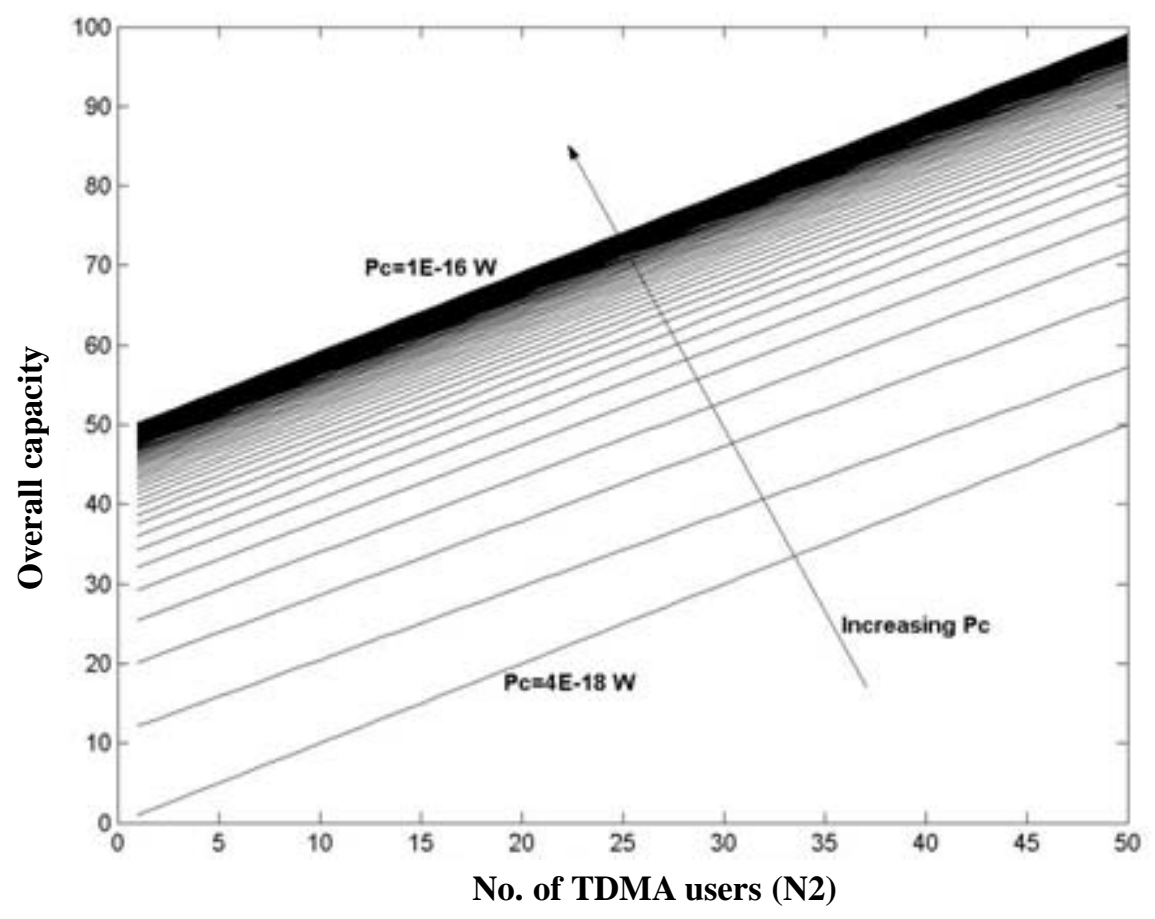

Figure 5. Variation of overall capacity with $N_{2}$ for different values of $\boldsymbol{P}_{\boldsymbol{c}}$

dation in the signal to noise ratio. If the amount of increase in the number of users cause this to be lower than the threshold then all users will be assumed in the off state and hence a sudden decrease in the capacity occurs. This suggests that it is of great importance to choose carefully the number of CDMA users in the hybrid system. The case is different when changing the number of TDMA users. Figure 5 shows variations of the overall capacity with the number of TDMA users $\left(N_{2}\right)$ for different values of $P_{c}$. It is obvious that the overall capacity increases with increasing $N_{2}$.
Depending on the above results, it is possible to say that under certain limits on values of $N_{1}, N_{2}, P_{c}$ and $P_{t}$, the hybrid system (CDMA+TDMA) can have a total capacity which is higher than the CDMA (or TDMA) system working alone by about $100 \%$. This conclusion can be explained with the help of Figs. 4 and 5. Maximum capacity with a TDMA system working alone, ie. $N_{1}=0$, or with a CDMA system working alone, ie. $N_{2}=0$, is 48 users for the parameter values assumed in this paper. While with the hybrid system maximum capacity can reach up to 96 users. This means that the spectral coexis- 
tence of CDMA and TDMA systems is possible and has real benefits under certain conditions.

\section{Conclusions}

In this paper, theoretical investigations on the spectral coexistence of narrow band CDMA and TDMA systems has been presented. Results of analysis have proved the possibility of the spectral coexistence of the two systems under certain conditions which shows that the available frequency band can be used in an efficient way. It has been shown that an increase of about $100 \%$ in the overall capacity can be obtained for a certain range of values for the number of CDMA users, number of TDMA users, received CDMA power, and received TDMA power.

\section{References}

Grieco, D. and Schilling, D., 1994, "The Capacity of Broadband CDMA Overlaying a GSM Cellular Systems," IEEE 44th Vehicular Technology Conference, Vol. 1, pp. 31-35.

Himimy, H.H. and Gupta, S.C., 1996, "Overlay of
Cellular CDMA or AMPS Forward and Reverse Link," IEEE Transactions on Vehicular Technology, Vol. 45(1), pp. 51-56.

Koorevaar, P. and Ruprecht, J., 1999, "Frequency Overlay of GSM and Cellular B-CDMA," IEEE Transaction on Vehicular Technology, Vol. 48(3), pp. 696-707.

Latchman, P. and Chitamu, P., 2003, " The Study of Band Sharing for GSM 900 and CDMA 2000 1X Coexistence within the $900 \mathrm{MHz}$ Cellular Band," www.ee.wits.ac.za / comms/.

Pabst, R. and Seidenberg, P., 2001, "Coexistence of UMTS and EGPRS to Enhance Network Capacity," Proc. of the 10th Aachen Symposium on Signal Theory, Germany, pp. 43-48.

Schilling, D., Lomp, G.R. and J. Garodnick, 1993, " Broadband CDMA Overlay," IEEE 43rd Vehicular Technology Conference, pp. 452-455.

Sinha, R., 1997, "Spread Spectrum Interference Issues in the $900 \mathrm{MHz}$ ISM Band," M.Sc. Thesis, the State University of New Jersey, USA.

Widdowson, T., 1997, "A CDMA Data Overlay of the GSM Network", IEEE - PIMRC, pp. 160-163. 